\title{
Ramsey Properties of Random Subgraphs of Pseudo-Random Graphs
}

\author{
Jia Shen* \\ Key Laboratory of Mathematics of Chinese Academy of Sciences \\ School of Mathematical Sciences \\ University of Science and Technology of China \\ Hefei, Anhui, China 230026 \\ jiajshen@gmail.com
}

Submitted: Oct 16, 2011; Accepted: Jun 22, 2012; Published: Jul 5, 2012

Mathematics Subject Classifications: 05C80, 05D10, 05D40

\begin{abstract}
Let $G=(V, E)$ be a $d$-regular graph of order $n$. Let $G_{p}$ be the random subgraph of $G$ for which each edge is selected from $E(G)$ independently at random with probability $p$. For a fixed graph $H$, define $m(H):=\max \left\{e\left(H^{\prime}\right) /\left(v\left(H^{\prime}\right)-1\right): H^{\prime} \subseteq H\right\}$. We prove that $n^{(m(H)-1) / m(H)} / d$ is a threshold function for $G_{p}$ to satisfy Ramsey, induced Ramsey, and canonical Ramsey properties with respect to vertex coloring, respectively, provided the eigenvalue $\lambda$ of $G$ that is second largest in absolute value is significantly smaller than $d$.

As a consequence, it is also shown that $n^{(m(H)-1) / m(H)} / d$ is a threshold function for $G_{p}$ to contain a family of vertex disjoint copies of $H$ (an $H$-packing) that covers $(1-o(1)) n$ vertices of $G$. Using a similar argument, the sharp threshold function for $G_{p}$ to contain $H$ as a subgraph is obtained as well.
\end{abstract}

Keywords: Random subgraph; pseudo-random; Ramsey property; $H$-packing;

\section{Introduction}

In this paper we study random subgraphs of pseudo-random graphs. Pseudo-random graphs, which have been intensively studied over the last two decades (see, e.g., [11]), are graphs whose edge distribution resembles closely that of a random graph with the same edge density. Let $G=(V, E)$ be a $d$-regular graph of order $n$, and $A$ the adjacency matrix of $G$. Let $\lambda_{1} \geqslant \cdots \geqslant \lambda_{n}$ be the eigenvalues of $A$. It follows from the Perron-Frobenius

\footnotetext{
* Research supported in part by NSFC grants 11101398 and 11026071 of China, and by the Fundamental Research Funds for the Central Universities of China.
} 
theorem that $\lambda_{1}=d$. Denote by $\lambda$ the eigenvalue of $A$ that is second largest in absolute value. Roughly speaking, a pseudo-random graph is a $d$-regular graph $G=(V, E)$ with $\lambda$ significantly smaller than $d$.

The classical Erdős-Rényi random graph model, namely the so-called binomial random graph $G(n, p)$, is formed by selecting each edge on a set of $n$ labeled vertices independently with probability $p$. Alternatively, $G(n, p)$ can be viewed as a random subgraph of the complete graph $K_{n}$. In the present paper, rather than studying a random subgraph of one particular graph $K_{n}$, we investigate random subgraphs of a graph $G$ from a broad class of regular graphs, known as pseudo-random graphs. The study of random subgraphs arising in this manner was initiated in [7].

Given a graph $G=(V, E)$ and an edge probability $0 \leqslant p=p(n) \leqslant 1$, the random subgraph $G_{p}$ of $G$ is formed by selecting each edge of $G$ independently with probability $p$.

Since the eigenvalues of $K_{n}$ are $n-1$ (multiplicity 1) and -1 (multiplicity $n-1$ ), $K_{n}$ is indeed a pseudo-random graph, by definition. Thus the random subgraph $G_{p}$ of pseudo-random graphs is a natural generalization of the classical Erdős-Rényi random graph $G(n, p)$, in that the host graph $G$ is from a much wider class of regular graphs including $K_{n}$.

For a graph property $\mathcal{P}$, say that $G_{p}$ satisfies $\mathcal{P}$ almost surely if the probability that $G_{p}$ satisfies $\mathcal{P}$ tends to 1 as $n$ tends to infinity. A function $t(n)$ is a threshold function for the property $\mathcal{P}$ if

$$
P\left(G_{p} \text { satisfies } \mathcal{P}\right) \rightarrow \begin{cases}0 & \text { if } p \ll t(n), \\ 1 & \text { if } p \gg t(n) .\end{cases}
$$

Some results concerning $G(n, p)$ can be naturally generalized to the random subgraph $G_{p}$ of pseudo-random graphs $G$. For example, the threshold function for the existence of a Hamilton cycle, and the appearance of a giant component were studied in Frieze et al. $[7,8]$. The threshold function for the connectivity of $G_{p}$ is obtained in [14].

Let $H$ be a fixed graph of order $h$. Given a graph $G=(V, E)$ of order $n$, a copy of $H$ in $G$ is a (not necessarily induced) subgraph $H^{\prime}$ of $G$ that is isomorphic to $H$. The arrow notation, introduced by Erdös and Rado, has proved particularly useful in Ramsey theory. We write $G \rightarrow(H)_{r}^{1}$ if, given any $r$-coloring of $V(G)$, there exists a copy of $H$ whose vertices are monochromatic. We determine the sharp threshold function for $G_{p} \rightarrow(H)_{r}^{1}$. Define

$$
m(H):=\max \left\{\frac{e\left(H^{\prime}\right)}{v\left(H^{\prime}\right)-1}: H^{\prime} \subseteq H\right\} .
$$

Theorem 1. Let $G=(V, E)$ be a d-regular graph of order $n$, and $\lambda$ the eigenvalue that is second largest in absolute value. Let $H$ be a fixed graph with maximum degree $\Delta$, and $m(H)$ defined as above. Suppose $d \gg \lambda^{1 / \Delta} n^{1-1 / \Delta}$. Then, for any integer $r \geqslant 2$,

(i) there exists a constant $c=c(H, r)>0$ such that if $p=c \frac{n^{(m(H)-1) / m(H)}}{d}$, then almost surely $G_{p} \nrightarrow(H)_{r}^{1}$; 
(ii) there exists a constant $C=C(H, r)>0$ such that if $p=C \frac{n^{(m(H)-1) / m(H)}}{d}$, then almost surely $G_{p} \rightarrow(H)_{r}^{1}$.

Remark 1. Note that the hypothesis $d \gg \lambda^{1 / \Delta(H)} n^{1-1 / \Delta(H)}$ guarantees $p=o(1)$, since $m(H) \leqslant \Delta(H) / 2$ yields $n^{(m(H)-1) / m(H)} / d \leqslant n^{1-2 / \Delta(H)} / d=o(1)$.

Remark 2. Clearly, if $d$ is small, then the thresholds in Theorem 1 to 5 may not occur. For example, if $d$ is small, then the host graph $G$ may not contain $H$ as a subgraph, so that a threshold does not exist in this case.

We now turn our attention to the induced Ramsey property of $G_{p}$. We write $G \stackrel{\text { ind }}{\rightarrow}(H)_{r}^{1}$ if given any $r$-coloring of the vertices $V(G)$ of $G$, there exists an induced copy of $H$ whose vertices are monochromatic.

Theorem 2. Let $G=(V, E)$ be a d-regular graph of order $n$, and $\lambda$ the eigenvalue that is second largest in absolute value. Let $H$ be a fixed graph with maximum degree $\Delta$, and $m(H)$ defined as above. Suppose $d \gg \lambda^{1 / \Delta} n^{1-1 / \Delta}$, and $d<\alpha n$, for some constant $\alpha<1$. Then, for any integer $r \geqslant 2$,

(i) there exists a constant $c=c(H, r)>0$ such that if $p=c \frac{n^{(m(H)-1) / m(H)}}{d}$, then almost surely $G_{p} \stackrel{\text { ind }}{\rightarrow}(H)_{r}^{1}$

(ii) there exists a constant $C=C(H, r)>0$ such that if $p=C \frac{n^{(m(H)-1) / m(H)}}{d}$, then almost surely $G_{p} \stackrel{\text { ind }}{\longrightarrow}(H)_{r}^{1}$.

Remark. For the classical Erdős-Rényi random graph $G(n, p)$, there does not exist a threshold function for the induced Ramsey property in general. This is one of the properties that indicate $G_{p}$ differs from the classical $G(n, p)$. We also see that the above requirement $d<\alpha n$, for some constant $\alpha<1$, is necessary.

Now we consider colorings on which no restriction (not even finiteness) is put on the number of colors used. Call a coloring $\chi$ of a set $S$ canonical if $\chi$ is either

(i) monochromatic [i.e., $\chi(s)=\chi(t)$ for all $s, t \in S$ ], or

(ii) distinct (or rainbow) [i.e., $\chi(s) \neq \chi(t)$ for all $s, t \in S, s \neq t$ ].

We write $G \rightarrow(H)_{c}^{1}$ to denote that for every coloring $\chi$ of $V(G)$ with an arbitrary number of colors, there exists a copy of $H$ on which the coloring $\chi$ is canonical. In other words, the vertices of this copy either all receive the same color or all receive distinct colors. Our next result shows that the threshold for $G \rightarrow(H)_{c}^{1}$ is the same as that for $G \rightarrow(H)_{r}^{1}$.

Theorem 3. Let $G=(V, E)$ be a d-regular graph of order $n$, and $\lambda$ the eigenvalue that is second largest in absolute value. Let $H$ be a graph with $e(H)>0, H \neq K_{2}$ or $K_{2} \cup K_{1}$, and $m(H)$ defined as above. Suppose $d \gg \lambda^{1 / \Delta} n^{1-1 / \Delta}$. Then, 
(i) there exists a constant $c=c(H, r)>0$ such that if $p=c \frac{n^{(m(H)-1) / m(H)}}{d}$, then almost surely $G_{p} \nrightarrow(H)_{c}^{1}$;

(ii) there exists a constant $C=C(H, r)>0$ such that if $p=C \frac{n^{(m(H)-1) / m(H)}}{d}$, then almost surely $G_{p} \rightarrow(H)_{c}^{1}$.

As a by-product, we also determine the threshold function for $H$-tiling. An $H$-tiling or $H$-packing in $G$ is a family of vertex-disjoint copies of $H$ in $G$. An $H$-factor of $G$, when $h$ divides $n$, is an $H$-packing $\mathcal{H}$ such that every vertex of $G$ is contained in a member of $\mathcal{H}$. Thus, $G$ has an $H$-factor if $G$ contains $n / h$ disjoint copies of $H$.

The objective of packing problems in graph theory is to find in $G$ as many disjoint copies of $H$ as possible. A fundamental result is the Hajnal-Szemerédi Theorem [9], which gives the minimum degree condition for a $K_{r}$-factor. Alon and Yuster [3] consider more general case, and obtain an asymptotic minimum degree condition for an $H$-factor. The reader is referred to [12] for a good survey. In the present paper, we also determine the sharp threshold function for $G_{p}$ to contain an almost $H$-factor, namely, an $H$-packing that covers $(1-o(1)) n$ vertices of $G$.

Theorem 4. Let $G=(V, E)$ be a d-regular graph of order $n$, and $\lambda$ the eigenvalue that is second largest in absolute value. Let $H$ be a fixed graph with maximum degree $\Delta$, and $m(H)$ defined as above. Suppose $d \gg \lambda^{1 / \Delta} n^{1-1 / \Delta}$. Then, for any $\epsilon>0$,

(i) there exists a constant $c=c(H, \epsilon)>0$ such that if $p=c \frac{n^{(m(H)-1) / m(H)}}{d}$, then almost surely $G_{p}$ does not contain an $H$-packing that covers all but at most $\epsilon$ vertices of $G$;

(ii) there exists a constant $C=C(H, \epsilon)>0$ such that if $p=C \frac{n^{(m(H)-1) / m(H)}}{d}$, then almost surely $G_{p}$ contains an $H$-packing that covers all but at most $\epsilon$ n vertices of $G$.

A related question, which turns out to be simpler than $H$-packing, concerns the threshold function for containing $H$ as a subgraph. Using a similar and simpler argument, we can also determine the threshold function for $G_{p}$ to contain a fixed $H$. Define

$$
m^{\prime}(H):=\max \left\{\frac{e\left(H^{\prime}\right)}{v\left(H^{\prime}\right)}: H^{\prime} \subseteq H\right\} .
$$

Theorem 5. Let $G=(V, E)$ be a d-regular graph of order $n$, and $\lambda$ the eigenvalue that is second largest in absolute value. Let $H$ be a fixed graph with maximum degree $\Delta$, and $m^{\prime}(H)$ defined as above. Suppose $d \gg \lambda^{1 / \Delta} n^{1-1 / \Delta}$. Then

(i) there exists a constant $c=c(H, \epsilon)>0$ such that if $p=c \frac{n^{\left(m^{\prime}(H)-1\right) / m^{\prime}(H)}}{d}$, then almost surely $G_{p}$ does not contain $H$ as a subgraph; 
(ii) there exists a constant $C=C(H, \epsilon)>0$ such that if $p=C \frac{n^{\left(m^{\prime}(H)-1\right) / m^{\prime}(H)}}{d}$, then almost surely $G_{p}$ contains $H$ as a subgraph.

Theorems 4 and 5 extend the celebrated results of Erdős and Rényi (cf. [4, 6]) on the threshold function for containing a fixed graph $H$ in the random graph $G(n, p)$.

\section{Preliminaries}

The proofs of our main results rely heavily on an inequality, due to Janson (cf. [1]), which estimates the probability that the sum of "mostly independent" random variables deviates from its mean. The inequality has proven especially useful when one seeks to show that the probability is bounded from above by a function that is exponentially small.

Let $\Omega$ be a finite universal set, and $R$ a random subset of $\Omega$, where $P(r \in R)=p_{r}$. So these events are mutually independent over $r \in \Omega$. Let $\left\{A_{i}: i \in I\right\}$ be subsets of $\Omega$, with $I$ a finite index set. Let $B_{i}$ be the event $A_{i} \subseteq R$, and $X_{i}$ the indicator random variable for $B_{i}$. Let $X:=\sum_{i \in I} X_{i}$ count the number of $A_{i} \subseteq R$. For $i, j \in I$, we write $i \sim j$ if $i \neq j$ and $A_{i} \cap A_{j} \neq \emptyset$. We define

$$
\Lambda:=\sum_{i \sim j} P\left(B_{i} \wedge B_{j}\right)
$$

where the sum is over ordered pairs. The Janson inequalities state that when the events $B_{i}$ are "mostly independent", $X$ is still close to Poisson distribution with mean $\mu=E(X)$, as is the case when $B_{i}$ 's are all independent.

Lemma 6 (Janson's Inequality). Let $B_{i}, \Lambda, \mu$ be as above, and assume that $P\left(B_{i}\right) \leqslant \gamma$ for all $i$. Then

$$
P(X=0) \leqslant \exp \left(-\mu+\frac{1}{1-\gamma} \frac{\Lambda}{2}\right) .
$$

Further, if $\Lambda \geqslant \mu(1-\gamma)$, then

$$
P(X=0) \leqslant \exp \left(\frac{-\mu^{2}(1-\gamma)}{2 \Lambda}\right)
$$

In a pseudo-random graph $G=(V, E)$, for $B, C \subset V$, let $e(B, C)$ denote the number of ordered pairs $(u, v)$ such that $u \in B, v \in C$ and $u v \in E(G)$; let $e(B)$ denote the number of edges in $G[B]$.

Note that subsets $B, C \subset V$ are not necessarily disjoint. If $B \cap C \neq \emptyset$, then by definition $e(B, C)$ counts the edges in $G[B \cap C]$ twice. Lemma 7 concerns edge distribution in a pseudo-random graph (cf. [1, Chap. 9] ).

Lemma 7. Suppose $B, C \subset V(G)$ are (not necessarily disjoint) subsets of vertices. Then

$$
\text { (1) }\left|e(B, C)-\frac{d}{n}\right| B|| C|| \leqslant \lambda \sqrt{|B||C|} \text {; }
$$


(2) $\left|e(B)-\frac{d}{n} \frac{|B|^{2}}{2}\right| \leqslant \frac{\lambda|B|}{2}$.

We also need Lemma 8, a result of Alon (cf. [11]) stating that, roughly speaking, every "large" subset of vertices in a pseudo-random graph contains about the same number of copies of $H$ as it "ought to" contain.

For a fixed graph $H$, denote by $\operatorname{Aut}(H)$ the automorphism group of $H$. Define $\operatorname{aut}(H):=|\operatorname{Aut}(H)|$.

Lemma 8. Let $H$ be a fixed graph with s vertices and $r$ edges, and maximum degree $\Delta(H)$. Let $G=(V, E)$ be a pseudo-random graph, and $\lambda$ the eigenvalue of $G$ that is second largest

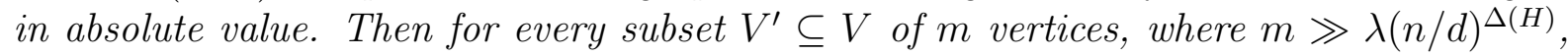
the number of (not necessarily induced) copies of $H$ in $G\left[V^{\prime}\right]$ is

$$
(1+o(1)) \frac{m^{s}}{\operatorname{aut}(H)}\left(\frac{d}{n}\right)^{r} .
$$

\section{Ramsey Property}

Lemma 9. for any $\epsilon>0$, there exists a constant $C=C(H, \epsilon)>0$ such that if $p=$ $C \frac{n^{(m(H)-1) / m(H)}}{d}$, then almost surely every subset of $\epsilon n$ vertices of $G_{p}$ contains a copy of $H$.

Proof. We use Janson's inequality to do so. Let $U \subseteq V$ be a subset of vertices of cardinality $\epsilon n$. Let $X$ denote the number of labeled copies of $H$ contained in $G_{p}[U]$, the subgraph induced by $U$ in $G_{p}$. By Lemma 8, the number of copies of $H$ contained in $G[U]$ is

$$
(1+o(1)) \frac{(\epsilon n)^{v}}{\operatorname{aut}(H)}\left(\frac{d}{n}\right)^{e} .
$$

For each of these copies, the probability that it is contained in $G_{p}[U]$ is $p^{e}$. Thus,

$$
\begin{aligned}
\mu=E(X) & =(1+o(1)) \frac{(\epsilon n)^{v}}{\operatorname{aut}(H)}\left(\frac{d}{n}\right)^{e} p^{e} \\
& =(1+o(1)) \frac{(\epsilon n)^{v}}{\operatorname{aut}(H)}\left(\frac{d}{n}\right)^{e}\left(C \frac{n^{(m(H)-1) / m(H)}}{d}\right)^{e} \\
& \sim \frac{\epsilon^{v} C^{e}}{\operatorname{aut}(H)} n^{v-e \frac{v^{\prime}-1}{e^{\prime}}} \\
& \geqslant \frac{\epsilon^{v} C^{e}}{\operatorname{aut}(H)} n^{v-(v-1)} \quad\left(\frac{v^{\prime}-1}{e^{\prime}} \leqslant \frac{v-1}{e}\right) \\
& =\frac{\epsilon^{v} C^{e}}{\operatorname{aut}(H)} n .
\end{aligned}
$$


Given two copies $H_{i}, H_{j}$ of $H$ in $G$, we write $i \sim j$ if $i \neq j$ and $E\left(H_{i}\right) \cap E\left(H_{j}\right) \neq \emptyset$. Let $H^{\prime \prime}$ be a subgraph of $H$ with $e\left(H^{\prime \prime}\right) \geqslant 1$. Denote by " $\simeq$ " the graph isomorphism. Then

$$
\begin{aligned}
\Lambda & :=\sum_{i \sim j} P\left(H_{i} \cup H_{j} \subseteq G_{p}\right)=\sum_{H^{\prime \prime} \subseteq H} \sum_{H_{i} \cap H_{j} \simeq H^{\prime \prime}} P\left(H_{i} \cup H_{j} \subseteq G_{p}\right) \\
& \leqslant \sum_{H^{\prime \prime} \subseteq H} \frac{(\epsilon n)^{v}}{\operatorname{aut}(H)}\left(\frac{d}{n}\right)^{e} p^{e} \cdot \frac{(\epsilon n)^{v-v^{\prime \prime}}}{\operatorname{aut}\left(H \backslash H^{\prime \prime}\right)}\left(\frac{d}{n}\right)^{e-e^{\prime \prime}} p^{e-e^{\prime \prime}} \\
& =\sum_{H^{\prime \prime} \subseteq H} \frac{(\epsilon n)^{2 v-v^{\prime \prime}}}{\operatorname{aut}(H) \operatorname{aut}\left(H \backslash H^{\prime \prime}\right)}\left(\frac{d}{n}\right)^{2 e-e^{\prime \prime}}\left(C \frac{n^{(m(H)-1) / m(H)}}{d}\right)^{2 e-e^{\prime \prime}} \\
& =\sum_{H^{\prime \prime} \subseteq H} \frac{\epsilon^{2 v-v^{\prime \prime}} C^{2 e-e^{\prime \prime}}}{\operatorname{aut}(H) \operatorname{aut}\left(H \backslash H^{\prime \prime}\right)} n^{2 v-v^{\prime \prime}-\frac{v^{\prime}-1}{e^{\prime}}\left(2 e-e^{\prime \prime}\right)} \\
& =: \sum_{H^{\prime \prime} \subseteq H} \Lambda\left(H^{\prime \prime}\right),
\end{aligned}
$$

where $\Lambda\left(H^{\prime \prime}\right)$ denotes the term in the sum corresponding to a fixed subgraph $H^{\prime \prime} \subseteq H$. Now

$$
\begin{aligned}
\frac{\mu^{2}}{\Lambda\left(H^{\prime \prime}\right)} & =\left(\frac{\epsilon^{v} C^{e}}{\operatorname{aut}(H)} n^{v-e / m(H)}\right)^{2} /\left(\frac{\epsilon^{2 v-v^{\prime \prime}} C^{2 e-e^{\prime \prime}}}{\operatorname{aut}(H) \operatorname{aut}\left(H \backslash H^{\prime \prime}\right)} n^{2 v-v^{\prime \prime}-\frac{1}{m(H)}\left(2 e-e^{\prime \prime}\right)}\right) \\
& =\frac{\epsilon^{v^{\prime \prime}} C^{e^{\prime \prime}} \operatorname{aut}\left(H \backslash H^{\prime \prime}\right)}{\operatorname{aut}(H)} n^{v^{\prime \prime}-\frac{v^{\prime}-1}{e^{\prime}} e^{\prime \prime}} \quad \quad\left(\frac{v^{\prime}-1}{e^{\prime}} \leqslant \frac{v^{\prime \prime}-1}{e^{\prime \prime}}\right) \\
& \geqslant \frac{\epsilon^{v^{\prime \prime}} C^{e^{\prime \prime}} \operatorname{aut}\left(H \backslash H^{\prime \prime}\right)}{\operatorname{aut}(H)} n^{v^{\prime \prime}-\left(v^{\prime \prime}-1\right)} \\
& \geqslant \frac{\epsilon^{v^{\prime \prime}} C^{e^{\prime \prime}} \operatorname{aut}\left(H \backslash H^{\prime \prime}\right)}{\operatorname{aut}(H)} n .
\end{aligned}
$$

Since there are at most $2^{\left(\begin{array}{l}v \\ 2\end{array}\right)-(v+1)}<2^{\left(\begin{array}{l}v \\ 2\end{array}\right)}$ subgraphs $H^{\prime \prime}$ in $H$ with $e\left(H^{\prime \prime}\right) \geqslant 1$, we see that

$$
\frac{\mu^{2}}{2 \Lambda} \geqslant \frac{\epsilon^{v^{\prime \prime}} C^{e^{\prime \prime}} \text { aut }\left(H \backslash H^{\prime \prime}\right)}{2^{\left(\begin{array}{l}
v \\
2
\end{array}\right)+1} \operatorname{aut}(H)} n>n,
$$

where $C$ can be large enough such that the last inequality holds.

Case 1. $\Lambda \leqslant \mu$. We know that $P\left(B_{i}\right)=P\left(H_{i} \subseteq G_{p}\right)=p^{e} \leqslant p=o(1)$, from the remark after Theorem 4 . Thus we can take $\gamma$ to be an arbitrarily small constant such that $P\left(B_{i}\right) \leqslant \gamma$. Also, note that

$$
\mu=E(X)=\frac{\epsilon^{v} C^{e}}{\operatorname{aut}(H)} n \geqslant \frac{\epsilon^{v^{\prime \prime}} C^{e^{\prime \prime}}}{\operatorname{aut}(H)} n \geqslant 2^{\left(\begin{array}{l}
v \\
2
\end{array}\right)+1} n>3 n .
$$

Thus

$$
P(X=0) \leqslant \exp (-\mu / 3)<\exp (-n)
$$


Case 2. $\Lambda>\mu$. Then, from (1) we see that

$$
P(X=0) \leqslant \exp \left(-\frac{\mu^{2}(1-\gamma)}{2 \Lambda}\right) \leqslant \exp (-(1-\gamma) n) .
$$

In either case, the probability that there is a subset of $\epsilon n$ vertices that contains no copy of $H$ is at most

$$
\begin{aligned}
\left(\begin{array}{c}
n \\
\epsilon n
\end{array}\right) P(X=0) & \leqslant\left(\frac{e n}{\epsilon n}\right)^{\epsilon n} \exp (-(1-\gamma) n) \\
& =\exp (\epsilon(1-\log \epsilon) n-(1-\gamma) n) \\
& \leqslant \exp (-n / 2)=o(1)
\end{aligned}
$$

since $\epsilon(1-\log \epsilon)=\epsilon-\epsilon \log \epsilon \rightarrow 0$ as $\epsilon \rightarrow 0$.

This shows that almost surely every subset of $\epsilon n$ vertices in $G_{p}$ contains a copy of $H$. Thus $G_{p}$ contains at least $(1-\epsilon) n / h$ vertex-disjoint copies of $H$.

Proof of Theorem 1. (i) We show that there exists a constant $C$ such that for $p=$ $C n^{(m(H)-1) / m(H)} / d, P\left(G_{p} \nrightarrow(H)_{r}^{1}\right)=o(1)$. Suppose $G_{p} \nrightarrow(H)_{r}^{1}$, namely there exists an $r$-coloring of $V(G)$ such that $G_{p}$ contains no monochromatic copy of $H$ in this coloring. Thus there exists a subset of $n / r$ vertices (the largest color class will do) that doesn't contain a copy of $H$. The probability of this latter event, by Lemma 9 , is $o(1)$.

(ii) Suppose $p=c n^{(m(H)-1) / m(H)} / d$, we show that

$$
P\left(G_{p} \rightarrow(H)_{r}^{1}\right)=o(1) .
$$

If $m(H):=\max \left\{e\left(H^{\prime}\right) /\left(v\left(H^{\prime}\right)-1\right): H^{\prime} \subseteq H\right\}=1$, then $H$ is a forest with $e(H)>0$. Since for $p=c / d, c<1, G_{p}$ almost surely consists of trees and unicyclic components (see [8, Theorem 2]), we see that $G_{p}$ can be properly colored with at most 3 colors so that all copies of $H$ are not monochromatic.

Now assume that $v(H) \geqslant 3$ and $m(H)>1$. Let $H_{1} \subseteq H$ be a smallest subgraph of $H$ with $e\left(H_{1}\right) /\left(v\left(H_{1}\right)-1\right)=m(H)>1$. Thus for any subgraph $H^{\prime} \subseteq H_{1}$, we have

$$
\frac{e\left(H^{\prime}\right)}{v\left(H^{\prime}\right)-1}<\frac{e\left(H_{1}\right)}{v\left(H_{1}\right)-1} .
$$

We construct a hypergraph $(V, \mathcal{E})$ whose vertex set is $V(G)$ and whose hyperedges are the vertex sets of copies of $H_{1}$ in $G_{p}$. Denote this hypergraph by $\mathcal{H}_{p}$. If $G_{p} \rightarrow(H)_{r}^{1}$, then $\chi\left(\mathcal{H}_{p}\right)>r$. We take an edgewise minimal subhypergraph $\mathcal{H}$ of $\mathcal{H}_{p}$ with the property $\chi(\mathcal{H})>r$.

Fact 1. For every hyperedge $E$ of $\mathcal{H}$, and every $v \in E$, there exists a hyperedge $E^{\prime} \in \mathcal{H}$ such that $E \cap E^{\prime}=\{v\}$, in other words, $E^{\prime}$ intersects $E$ only in $v$.

If $\mathcal{H}$ is edge-minimal with $\chi(\mathcal{H}) \geqslant r+1$, then for any $E \in \mathcal{H}, \mathcal{H}-E$ admits a proper $r$ coloring. In any such coloring, vertices of $E$ must be monochromatic, as otherwise those 
vertices receive different colors, then the $r$-coloring is a proper coloring of $\mathcal{H}$, contrary to the hypothesis that $\chi(\mathcal{H})>r$.

Suppose that in a hyperedge $E$, there exists a vertex $v$ such that for any other hyperedge $E^{\prime} \in \mathcal{H}, E^{\prime} \cap E \neq\{v\}$. Thus $E^{\prime} \cap E$ either doesn't involve $v$ at all or, if it does, $E^{\prime} \cap E$ contains at least two vertices, one of which is $v$. Now, $\mathcal{H}-E$ admits a proper $r$-coloring in which vertices in $E$ all receive the same color. Recolor the vertex $v$ with a different color (that has been used, not new), then $E$ receives two colors; for every $E^{\prime} \in \mathcal{H}$, if $E^{\prime}$ doesn't contain $v$ then colors of vertices in $E^{\prime}$ do not change; if $E^{\prime}$ contains $v$ then, by assumption, $E^{\prime}$ contains another vertex $u$ in $E$, which receives a color different from that of $v$ in this modified coloring.

Thus we see that $\mathcal{H}$ can be properly $r$-colored, contrary to the hypothesis that $\mathcal{H}$ is edge-minimal with $\chi(\mathcal{H}) \geqslant r+1$. Hence Fact 1 is proven.

Say that a sequence $E_{1}, E_{2}, \ldots, E_{k}$ of hyperedges forms a linear path in the hypergraph $\mathcal{H}$ if consecutive hyperedges intersect in exactly one vertex, i.e.,

$$
\left|E_{i} \cap E_{j}\right|= \begin{cases}1 & \text { if }|j-i|=1, \\ 0 & \text { otherwise. }\end{cases}
$$

Now let $P$ be a longest linear path in $\mathcal{H}$. (The reason that we require the path to be linear is because then the containment of these copies of $H_{1}$ is independent, and the probability of a hyperedge in the path $P$ is $p^{e\left(H_{1}\right)}$.)

It follows from Fact 1 that $P$ has at least two hyperedges $E_{1}$ and $E_{2}$. Suppose $x$ and $y$ are two vertices that are contained only in the first hyperedge $E_{1}$ of $P$. Fact 1 yields two hyperedges $E_{x}$ and $E_{y}$ that intersect $E_{1}$ and $E_{2}$ only in $x$ and $y$, respectively.

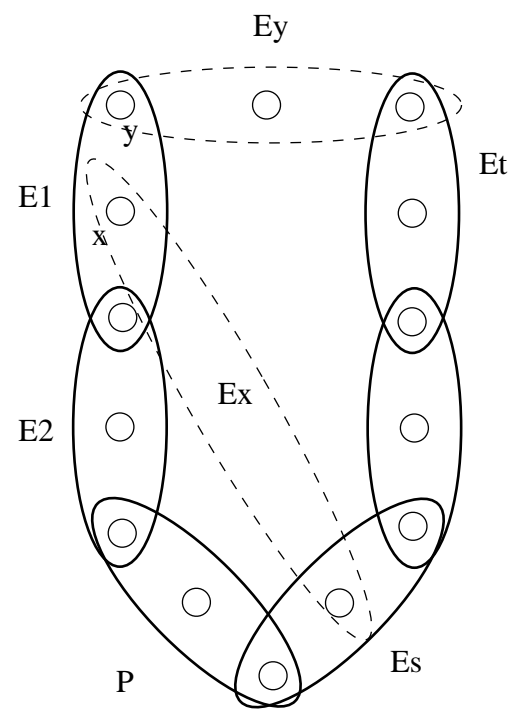

Figure 1: Linear path $P$ and hyperedges $E_{x}, E_{y}$

We see from the maximality of $P$ that $\left|E_{x} \cap P\right| \geqslant 2$ and $\left|E_{y} \cap P\right| \geqslant 2$. It follows that there exists a hyperedge in $P$ which intersects $E_{x}$, and which is different from $E_{1}$. Let $E_{s}$ 
denote the first hyperedge in $P$ such that $E_{s}$ intersects $E_{x}$, and $E_{s} \neq E_{1}$. Similarly, let $E_{t}$ denote the first hyperedge in $P$ such that $E_{t}$ intersects $E_{y}$, and $E_{t} \neq E_{2}$, as is depicted in Figure 1.

Without loss of generality, we assume $s \leqslant t$. Thus, $P \cup E_{x}$ is a cycle $E_{1}, E_{2}, \ldots, E_{s}, E_{x}$, where the first $s$ hyperedges $E_{1}, E_{2}, \ldots, E_{s}$ form a linear path. Similarly, $P \cup E_{y}$ is a cycle $E_{1}, E_{2}, \ldots, E_{t}, E_{y}$, where the first $t$ hyperedges $E_{1}, E_{2}, \ldots, E_{t}$ form a linear path, as indicated in Figure 1. We consider the events

$$
A_{\ell}: \quad\left|E_{y} \cap E_{t}\right|=\ell, \quad \ell=1,2, \ldots, h_{1}-1
$$

Then

$$
P\left(G_{p} \rightarrow(H)_{r}^{1}\right) \leqslant P(\chi(\mathcal{H})>r) \leqslant P\left(\bigvee_{\ell} A_{\ell}\right)
$$

Observe that the event $A_{\ell}, \ell \geqslant 2$, implies that there exists a hypercycle $\left(E_{1}, E_{2}, \ldots, E_{t}, E_{y}\right)$, $t \geqslant 3$, for which the first $t$ hyperedges form a linear path, and $H^{\prime}:=E_{y} \cap E_{t}\left(\left|H^{\prime}\right|=\ell\right)$ spans a proper subgraph of $H$. Hence, by Lemma 8,

$$
\begin{aligned}
P\left(\bigvee_{\ell \geqslant 2} A_{\ell}\right) & \leqslant \sum_{t \geqslant 3} \sum_{H^{\prime} \subsetneq H_{1}} \frac{n^{t\left(v\left(H_{1}\right)-1\right)-\left(v\left(H^{\prime}\right)-1\right)}}{\operatorname{aut}\left(P \cup E_{y}\right)}\left(\frac{d}{n}\right)^{t e\left(H_{1}\right)-e\left(H^{\prime}\right)} p^{t e\left(H_{1}\right)-e\left(H^{\prime}\right)} \\
& \leqslant \sum_{t} \sum_{H^{\prime} \subsetneq H_{1}}\left(n^{\left(v\left(H_{1}\right)-1\right) / e\left(H_{1}\right)} c n^{-1 / m(H)}\right)^{t e\left(H_{1}\right)} n^{-\left(\left(v\left(H^{\prime}\right)-1\right)-e\left(H^{\prime}\right) / m(H)\right)} c^{-e\left(H^{\prime}\right)} \\
& \leqslant \sum_{t} \sum_{H^{\prime} \subsetneq H_{1}} c^{t e\left(H_{1}\right)} n^{-\delta} c^{-e\left(H^{\prime}\right)} \\
& \leqslant 2^{h} c^{-h} \sum_{t}\left(c^{e\left(H_{1}\right)}\right)^{t} n^{-\delta} \\
& =o(1)
\end{aligned}
$$

where $\delta:=\left(\left(v\left(H^{\prime}\right)-1\right) / e\left(H^{\prime}\right)-\left(v\left(H_{1}\right)-1\right) / e\left(H_{1}\right)\right) e\left(H^{\prime}\right)>0$.

Thus we see that the hypercycle formed by the hyperedges $E_{1}, E_{2}, \ldots, E_{t}, E_{y}$ is almost surely a linear cycle, namely consecutive hyperedges intersect in exactly one vertex, including cyclically. Now we estimate $P\left(A_{1}\right)$. Let $B_{1}$ and $B_{2}$ denote the events that $A_{1}$ holds with $t \geqslant B \log n$ and $t<B \log n$, respectively. By Lemma 8,

$$
\begin{aligned}
P\left(B_{1}\right) & \leqslant \sum_{t \geqslant B \log n} \frac{n^{t\left(v\left(H_{1}\right)-1\right)+1}}{\operatorname{aut}(P)}\left(\frac{d}{n}\right)^{t e\left(H_{1}\right)} p^{t e\left(H_{1}\right)} \\
& \leqslant \sum_{t \geqslant B \log n} n^{t\left(v\left(H_{1}\right)-1\right)+1}(c n)^{-t e\left(H_{1}\right) / m(H)} \\
& =n \sum_{t \geqslant B \log n}\left(c^{e\left(H_{1}\right)}\right)^{t} \\
& =o(1) .
\end{aligned}
$$


For the probability of $B_{2}$, we observe that in the linear cycle $\left(E_{1}, E_{2}, \ldots, E_{t}, E_{y}\right), E_{x}$ intersects some hyperedge $E_{s}$ and $H^{\prime}:=E_{x} \cap E_{s}$ spans a proper subgraph of $H$. For a fixed $H^{\prime}=E_{x} \cap E_{s}$, the probability that $G_{p}$ contains the hyperedges $E_{1}, E_{2}, \ldots, E_{t}, E_{y}, E_{x}$ is, by Lemma 8 ,

$$
\frac{n^{(t+1)\left(v\left(H_{1}\right)-1\right)-\left(v\left(H^{\prime}\right)-1\right)}}{\operatorname{aut}\left(P \cup E_{x} \cup E_{y}\right)}\left(\frac{d}{n}\right)^{(t+1) e\left(H_{1}\right)-e\left(H^{\prime}\right)} p^{(t+1) e\left(H_{1}\right)-e\left(H^{\prime}\right)}
$$

Note that there are at most $(h B \log n)^{v\left(H^{\prime}\right)}$ ways to embed $H^{\prime}=E_{x} \cap E_{s}$ in the linear cycle $\left(E_{1}, E_{2}, \ldots, E_{t}, E_{y}\right)$. Hence, using Lemma 8, we have

$$
\begin{aligned}
P\left(B_{2}\right) \leqslant & \sum_{t=3}^{B \log n} \sum_{H^{\prime} \subseteq H_{1}}(h B \log n)^{v\left(H^{\prime}\right)} \frac{n^{(t+1)\left(v\left(H_{1}\right)-1\right)-\left(v\left(H^{\prime}\right)-1\right)}}{\operatorname{aut}\left(P \cup E_{x} \cup E_{y}\right)}\left(\frac{d}{n} p\right)^{(t+1) e\left(H_{1}\right)-e\left(H^{\prime}\right)} \\
\leqslant & c^{-h} \sum_{t=3}^{B \log n} \sum_{H^{\prime} \subseteq H_{1}}(h B \log n)^{v\left(H^{\prime}\right)}\left(c^{e\left(H_{1}\right)}\right)^{t+1}\left(n^{\left(v\left(H_{1}\right)-1\right) / e\left(H_{1}\right)-1 / m(H)}\right)^{(t+1) e\left(H_{1}\right)} \\
& \times\left(n^{\left(v\left(H^{\prime}\right)-1\right) / e\left(H^{\prime}\right)-1 / m(H)}\right)^{-e\left(H^{\prime}\right)} \\
\leqslant & (h B)^{h} c^{-h} 2^{h} \sum_{t=3}^{B \log n}(\log n)^{v\left(H^{\prime}\right)}\left(c^{e\left(H_{1}\right)}\right)^{t+1} n^{-\delta} \\
= & O\left((\log n)^{h+1} n^{-\delta}\right)=o(1),
\end{aligned}
$$

where $\delta:=\left(\left(v\left(H^{\prime}\right)-1\right) / e\left(H^{\prime}\right)-\left(v\left(H_{1}\right)-1\right) / e\left(H_{1}\right)\right) e\left(H^{\prime}\right)>0$. Thus, we see that $P\left(\bigvee_{\ell \geqslant 1} A_{\ell}\right)=o(1)$, and hence $P\left(G_{p} \rightarrow(H)_{r}^{1}\right)=o(1)$.

\section{Induced Ramsey Property}

Proof of Theorem 2. (i) By Theorem 1 (i), if $p=c \frac{n^{(m(H)-1) / m(H)}}{d}, c$ sufficiently small, then almost surely there is an $r$-coloring of $V\left(G_{p}\right)$ such that all copies of $H$ receive at least two colors, which implies that induced copies of $H$ all receive at least two colors.

(ii) We estimate $P\left(G_{p} \stackrel{\text { ind }}{\longrightarrow}(H)_{r}^{1}\right)$. Suppose that there is an $r$-coloring of $V(G)$ such that each induced copy of $H$ receives at least 2 colors. Then there exists a set of $n / r$ vertices containing no induced copies of $H$ (the largest color class will do).

In [11], it is also shown that if the hypotheses in Lemma 8 all hold true, and

1. $d=o(n)$, then the number of induced copies of $H$ in $G$ is $(1+o(1)) \frac{n^{s}}{\operatorname{aut}(H)}\left(\frac{d}{n}\right)^{r}$;

2. if $d / n<\alpha$, for a constant $0<\alpha<1$, then the number of induced copies of $H$ in $G$ is $\frac{\Omega\left(n^{s}\right)}{\operatorname{aut}(H)}\left(\frac{d}{n}\right)^{r}$. 
Using this result, along with Janson's inequality, we can show an analog of Lemma 9 as follows. Let $U \subseteq V(G)$ be a subset of $V(G)$ of cardinality $n / r$. Let $X$ be the induced copies of $H$ in $G_{p}[U]$. Proceeding in much the same way as the proof of Lemma 9, and using similar calculations, we can show that $E(X)=\Omega(n)$ and $\mu^{2} / 2 \Lambda>n$. Thus the probability that a set of $n / r$ vertices contains no induced copies of $H$ is bounded above by $\left(\begin{array}{c}n \\ n / r\end{array}\right) \exp (-n)=o(1)$.

Remark. For the classical Erdős-Rényi random graph $G(n, p)$, there does not exist a threshold for the induced Ramsey property in general. A fundamental reason for this is monotonicity. The complete graph (or a sufficiently dense pseudo-random graph) will not contain $H$ as an induced subgraph. Thus threshold properties for induced copies of $H$ occur for random subgraphs of sparse pseudo-random graphs, but not for random subgraphs of graphs with high linear degree.

\section{Canonical Ramsey Property}

In this section we prove Theorem 3. The proof of Theorem 3 relies on Proposition 10, which counts the number of copies of $H$ for which each vertex is from a given subset $V_{i}$ of vertices in a pseudo-random graph $G$. This result is a generalization of Lemma 8, and the proof follows from the same idea as that of Lemma 8 (cf. [11]).

Proposition 10. Let $H$ be a fixed graph on vertices $\left\{v_{1}, \ldots, v_{h}\right\}$ and with $r$ edges, and maximum degree $\Delta(H)$, where $h, r$ and $\Delta(H)$ are fixed values. Let $G=(V, E)$ be a pseudo-random graph. Then for subsets $V_{1}, \ldots, V_{h} \subseteq V$, where $m_{i}=\left|V_{i}\right| \gg \lambda(n / d)^{\Delta(H)}$, $1 \leqslant i \leqslant h$, the number of (not necessarily induced) copies of $H$ with $v_{i}$ sent into $V_{i}$, is

$$
(1+o(1)) \frac{m_{1} m_{2} \ldots m_{h}}{\operatorname{aut}(H)}\left(\frac{d}{n}\right)^{r} \text {, as } n \rightarrow \infty .
$$

Proof. We consider a random mapping from $V(H)$ to $V(G)$ that sends vertex $v_{i}$ to subset $V_{i}$, for all $i=1,2, \ldots, h$. Denote by $A(H)$ the event that every edge of $H$ is mapped to an edge of $G$. In such a case we say that the mapping is an embedding of $H$ into $G$. It suffices for our purposes to show

$$
P(A(H))=(1+o(1))\left(\frac{d}{n}\right)^{r}
$$

We prove (2) by induction on the number $r$ of edges. If $r=0$, clearly the assertion holds. Suppose that (2) holds for all graphs with at most $r-1$ edges. For an edge $u v \in E(G)$, let $H_{u v}:=H-u v$ denote the subgraph of $H$ on the vertex set $V(H)$ with the edge $u v$ deleted. Let $H_{u}:=H-u, H_{v}=H-v$, and $H^{\prime}:=H-\{u, v\}$. Write $r^{\prime}$ for the number $e\left(H^{\prime}\right)$ of edges of $H^{\prime}$, and note that

$$
r-r^{\prime} \leqslant 2(\Delta(H)-1)+1=2 \Delta(H)-1 .
$$

THE ELECTRONiC Journal of COMBinatorics 19(3) (2012), \#P2 
By the induction hypothesis,

$$
P\left(A\left(H^{\prime}\right)\right)=(1+o(1))\left(\frac{d}{n}\right)^{r^{\prime}}
$$

Thus, in order to get $P(A(H))$, we need only find $P\left(A(H) \mid A\left(H^{\prime}\right)\right)$. We first find $P\left(A\left(H_{u v}\right) \mid A\left(H^{\prime}\right)\right)$. For an embedding $f^{\prime}$ of $H^{\prime}$, since $V\left(H^{\prime}\right)=h-2$, there are two sets $V_{\alpha}, V_{\beta}$ (depending on $f^{\prime}$, of course) among $V_{1}, \ldots, V_{h}$ that do not contain images of vertices in $V\left(H^{\prime}\right)$. Denote these two sets $V_{\alpha}, V_{\beta}$ by $W_{1}=W_{1}\left(f^{\prime}\right)$ and $W_{2}=W_{2}\left(f^{\prime}\right)$. Denote by $\nu_{i}\left(u, f^{\prime}\right)$ (resp. $\left.\nu_{i}\left(v, f^{\prime}\right)\right)$ the number of extensions of $f^{\prime}$ to an embedding of $H_{v}=H-v\left(\operatorname{resp} . H_{u}=H-u\right)$ in $V$ with $u($ resp. $v)$ sent to $W_{i}, i=1,2$. Since the number of extensions of $f^{\prime}$ to an embedding of $H_{u v}$ in $V^{\prime}$ is $\nu_{1}\left(u, f^{\prime}\right) \nu_{2}\left(v, f^{\prime}\right)+\nu_{2}\left(u, f^{\prime}\right) \nu_{1}\left(v, f^{\prime}\right)$, we have

$$
P\left(A\left(H_{u v}\right) \mid f^{\prime}\right)=\frac{\nu_{1}\left(u, f^{\prime}\right) \nu_{2}\left(v, f^{\prime}\right)+\nu_{2}\left(u, f^{\prime}\right) \nu_{1}\left(v, f^{\prime}\right)}{\left|W_{1}\right|\left|W_{2}\right|+\left|W_{2}\right|\left|W_{1}\right|} .
$$

Taking expectation over all embeddings $f^{\prime}$, (4) yields

$$
\begin{aligned}
P\left(A\left(H_{u v}\right) \mid A\left(H^{\prime}\right)\right) & =\sum_{f^{\prime}} P\left(A\left(H_{u v}\right) \mid f^{\prime}\right) P\left(f^{\prime} \mid A\left(H^{\prime}\right)\right) \\
& =\sum_{f^{\prime}} \frac{\nu_{1}\left(u, f^{\prime}\right) \nu_{2}\left(v, f^{\prime}\right)+\nu_{2}\left(u, f^{\prime}\right) \nu_{1}\left(v, f^{\prime}\right)}{\left|W_{1}\right|\left|W_{2}\right|+\left|W_{2}\right|\left|W_{1}\right|} P\left(f^{\prime} \mid A\left(H^{\prime}\right)\right) \\
& =E_{f^{\prime}}\left(\frac{\nu_{1}\left(u, f^{\prime}\right) \nu_{2}\left(v, f^{\prime}\right)+\nu_{2}\left(u, f^{\prime}\right) \nu_{1}\left(v, f^{\prime}\right)}{\left|W_{1}\left(f^{\prime}\right)\right|\left|W_{2}\left(f^{\prime}\right)\right|+\left|W_{2}\left(f^{\prime}\right)\right|\left|W_{1}\left(f^{\prime}\right)\right|} \mid A\left(H^{\prime}\right)\right) .
\end{aligned}
$$

On the other hand,

$$
P\left(A\left(H_{u v}\right)=P\left(A\left(H_{u v} \mid A\left(H^{\prime}\right)\right) P\left(A\left(H^{\prime}\right)\right),\right.\right.
$$

and by the induction hypothesis, $P\left(A\left(H_{u v}\right)\right)=(1+o(1))\left(\frac{d}{n}\right)^{r-1}$ and $P\left(A\left(H^{\prime}\right)\right)=(1+$ $o(1))\left(\frac{d}{n}\right)^{r^{\prime}}$. Substituting these quantities in $(6)$, we get

$$
P\left(A\left(H_{u v} \mid A\left(H^{\prime}\right)\right)=(1+o(1))\left(\frac{d}{n}\right)^{r-1-r^{\prime}}\right.
$$

From (5) and (7), we have

$$
E_{f^{\prime}}\left(\frac{\nu_{1}\left(u, f^{\prime}\right) \nu_{2}\left(v, f^{\prime}\right)+\nu_{2}\left(u, f^{\prime}\right) \nu_{1}\left(v, f^{\prime}\right)}{2\left|W_{1}\left(f^{\prime}\right)\right|\left|W_{2}\left(f^{\prime}\right)\right|} \mid A\left(H^{\prime}\right)\right)=(1+o(1))\left(\frac{d}{n}\right)^{r-1-r^{\prime}}
$$

Now let $f$ be a random one-to-one mapping from $V(H)$ into $V^{\prime}$. Let $f^{\prime}$ be a fixed embedding of $H^{\prime}$. Then

$$
P\left(A(H)|f|_{H^{\prime}}=f^{\prime}\right)=\frac{d}{n} \frac{\nu_{1}\left(u, f^{\prime}\right) \nu_{2}\left(v, f^{\prime}\right)+\nu_{2}\left(u, f^{\prime}\right) \nu_{1}\left(v, f^{\prime}\right)}{\left|W_{1}\left(f^{\prime}\right)\right|\left|W_{2}\left(f^{\prime}\right)\right|+\left|W_{2}\left(f^{\prime}\right)\right|\left|W_{1}\left(f^{\prime}\right)\right|}+\delta_{1}+\delta_{2},
$$


where $\left|\delta_{i}\right| \leqslant \lambda \frac{\sqrt{\nu_{i}\left(u, f^{\prime}\right) \nu_{3-i}\left(v, f^{\prime}\right)}}{2\left|W_{1}\left(f^{\prime}\right)\right|\left|W_{2}\left(f^{\prime}\right)\right|}, i=1,2$. This follows from Lemma 7 , where we take the possible images of $u$ as the set $B$ and the possible images of $v$ as the set $C$, or the other way around. Averaging over embeddings $f^{\prime}$ we get

$$
\begin{aligned}
P\left(A(H) \mid A\left(H^{\prime}\right)\right)= & \sum_{f^{\prime}} P\left(A(H) \mid f^{\prime}\right) P\left(f^{\prime} \mid A\left(H^{\prime}\right)\right) \\
= & \sum_{f^{\prime}}\left(\frac{d}{n} \frac{\nu_{1}\left(u, f^{\prime}\right) \nu_{2}\left(v, f^{\prime}\right)+\nu_{2}\left(u, f^{\prime}\right) \nu_{1}\left(v, f^{\prime}\right)}{\left|W_{1}\left(f^{\prime}\right)\right|\left|W_{2}\left(f^{\prime}\right)\right|+\left|W_{2}\left(f^{\prime}\right)\right|\left|W_{1}\left(f^{\prime}\right)\right|}+\delta_{1}+\delta_{2}\right) P\left(f^{\prime} \mid A\left(H^{\prime}\right)\right) \\
= & \frac{d}{n} E_{f^{\prime}}\left(\frac{\nu_{1}\left(u, f^{\prime}\right) \nu_{2}\left(v, f^{\prime}\right)+\nu_{2}\left(u, f^{\prime}\right) \nu_{1}\left(v, f^{\prime}\right)}{2\left|W_{1}\left(f^{\prime}\right)\right|\left|W_{2}\left(f^{\prime}\right)\right|} \mid A\left(H^{\prime}\right)\right) \\
& +E_{f^{\prime}}\left(\delta_{1}+\delta_{2} \mid A\left(H^{\prime}\right)\right) \\
= & (1+o(1)) \frac{d}{n}\left(\frac{d}{n}\right)^{r-1-r^{\prime}}+E_{f^{\prime}}\left(\delta_{1}+\delta_{2} \mid A\left(H^{\prime}\right)\right) .
\end{aligned}
$$

By Jensen's inequality,

$$
\begin{aligned}
\left|E_{f^{\prime}}\left(\delta_{1} \mid A\left(H^{\prime}\right)\right)\right| & \leqslant E_{f^{\prime}}\left(\lambda \frac{\sqrt{\nu_{1}\left(u, f^{\prime}\right) \nu_{2}\left(v, f^{\prime}\right)}}{2\left|W_{1}\left(f^{\prime}\right)\right|\left|W_{2}\left(f^{\prime}\right)\right|} \mid A\left(H^{\prime}\right)\right) \\
& \leqslant \frac{\lambda}{\sqrt{2\left|W_{1}\left(f^{\prime}\right)\right|\left|W_{2}\left(f^{\prime}\right)\right|}} E_{f^{\prime}}\left(\sqrt{\frac{\nu_{1}\left(u, f^{\prime}\right) \nu_{2}\left(v, f^{\prime}\right)}{2\left|W_{1}\left(f^{\prime}\right)\right|\left|W_{2}\left(f^{\prime}\right)\right|}} \mid A\left(H^{\prime}\right)\right) \\
& \leqslant \frac{\lambda}{\sqrt{2} m} \sqrt{E_{f^{\prime}}\left(\frac{\nu_{1}\left(u, f^{\prime}\right) \nu_{2}\left(v, f^{\prime}\right)}{2\left|W_{1}\left(f^{\prime}\right)\right|\left|W_{2}\left(f^{\prime}\right)\right|} \mid A\left(H^{\prime}\right)\right)} \\
& \leqslant(1+o(1)) \frac{\lambda}{\sqrt{2} m}\left(\frac{d}{n}\right)^{\left(r-1-r^{\prime}\right) / 2}
\end{aligned}
$$

where $m:=\min \left\{m_{1}, \ldots, m_{s}\right\}$. Analogously,

$$
\left|E_{f^{\prime}}\left(\delta_{2} \mid A\left(H^{\prime}\right)\right)\right| \leqslant(1+o(1)) \frac{\lambda}{\sqrt{2} m}\left(\frac{d}{n}\right)^{\left(r-1-r^{\prime}\right) / 2} .
$$

Thus

$$
\begin{aligned}
P\left(A(H) \mid A\left(H^{\prime}\right)\right) & =(1+o(1))\left(\frac{d}{n}\right)^{r-r^{\prime}}\left(1+\frac{\sqrt{2} \lambda}{m}\left(\frac{d}{n}\right)^{\left(r-1-r^{\prime}\right) / 2-\left(r-r^{\prime}\right)}\right) \\
& =(1+o(1))\left(\frac{d}{n}\right)^{r-r^{\prime}}\left(1+\frac{\sqrt{2} \lambda}{m}\left(\frac{n}{d}\right)^{\left(r-r^{\prime}+1\right) / 2}\right) \\
& \leqslant(1+o(1))\left(\frac{d}{n}\right)^{r-r^{\prime}}\left(1+\frac{\sqrt{2} \lambda}{m}\left(\frac{n}{d}\right)^{\Delta(H)}\right) \\
& =(1+o(1))\left(\frac{d}{n}\right)^{r-r^{\prime}},
\end{aligned}
$$


by our assumptions on the parameters.

Thus

$$
P(A(H))=P\left(A(H) \mid A\left(H^{\prime}\right)\right) P\left(A\left(H^{\prime}\right)\right)=(1+o(1))\left(\frac{d}{n}\right)^{r} .
$$

Remark. The subsets $V_{1}, V_{2}, \ldots, V_{h}$ are not necessarily disjoint. In particular, if we take $V_{1}=V_{2}=\cdots=V_{h}$ in Proposition 10, then we get Lemma 8.

Proof of Theorem 3. (i) If $v(H) \geqslant 4$ then from Theorem 1 we know there exists $c>0$ such that for $p=c \frac{n^{(m(H)-1) / m(H)}}{d}$, almost surely $G_{p} \nrightarrow(H)_{3}^{1}$, that is, $G_{p}$ admits a 3-coloring in which there is no monochromatic copy of $H$. Since $v(H) \geqslant 4$, in this same coloring copies of $H$ cannot be rainbow either. Thus we see that there exists a coloring of $V(G)$ such that in the coloring there is no monochromatic copy of $H$, nor does $G_{p}$ contain rainbow copy of $H$ in this coloring.

Similarly, if $H=K_{3}$ or $P_{3}$, the 3-vertex path, Theorem 1 (ii) implies that there exists $c>0$ such that for $p=c \frac{n^{(m(H)-1) / m(H)}}{d}$, almost surely $G_{p} \nrightarrow(H)_{2}^{1}$, that is, $G_{p}$ admits a 2 -coloring in which there is no monochromatic copy of $H$. Since $v(H)=3$, in this same coloring copies of $H$ cannot be rainbow either. Thus we see that there exists a coloring of $V(G)$ such that in the coloring there is no monochromatic copy of $H$, nor does $G_{p}$ contain rainbow copy of $H$ in this coloring.

(ii) Given a coloring of $V(G)$, if some color is used at least $n / 2 h$ times, then there exists a color class of size at least $n / 2 h$. By Lemma 9 , we know that in this coloring, $G_{p}$ contains a monochromatic copy of $H$.

Henceforth we assume that each color is used at most $n / 2 h$ times. In this case we show that $G_{p}$ almost surely contains a rainbow copy of $H$ in the coloring.

Since each color class is of size at most $n / 2 h$, there exist $h$ disjoint subsets of vertices $V_{1}, \ldots, V_{h}$, each of size $n / 2 h \leqslant\left|V_{i}\right| \leqslant n / h, 1 \leqslant i \leqslant h$, such that none of the colors is present in more than one subset $V_{i}$. (Such subsets can be obtained by partitioning $V(G)$ according to the color classes.) Let $A$ be the set of mappings $\alpha: V(H) \rightarrow V(G)$ such that $\alpha\left(v_{i}\right) \in V_{i}, 1 \leqslant i \leqslant h$. Set $A_{\alpha}:=\left\{\alpha\left(v_{i}\right) \alpha\left(v_{j}\right): v_{i} v_{j} \in E(G)\right\}$. Let $B_{\alpha}$ be the event that $A_{\alpha}$ is contained in $G_{p}$, and $X_{\alpha}$ the corresponding indicator random variable. Define $X:=\sum_{\alpha \in A} X_{\alpha}$, and $\Lambda:=\sum_{i \sim j} P\left(A_{i} \wedge A_{j}\right)$. Clearly, $P\left(B_{\alpha}\right)=p^{e} \leqslant p=o(1)$.

Using Proposition 10, we have

$$
\begin{aligned}
\mu=E(X) & \geqslant \frac{m_{1} \ldots m_{h}}{\operatorname{aut}(H)}\left(\frac{d}{n}\right)^{e(H)} p^{e(H)} \\
& \geqslant\left(\frac{n}{2 h}\right)^{v(H)}\left(\frac{d}{n}\right)^{e(H)} p^{e(H)},
\end{aligned}
$$


and

$$
\begin{aligned}
\Lambda & \leqslant \sum_{H^{\prime} \subseteq H, e(H)>0}\left(\frac{n}{h}\right)^{2 v(H)-v\left(H^{\prime}\right)}\left(\frac{d}{n}\right)^{2 e(H)-e\left(H^{\prime}\right)} p^{2 e(H)-e\left(H^{\prime}\right)} \\
& =2^{h} h^{v\left(H^{\prime}\right)} n^{-v\left(H^{\prime}\right)}\left(C n^{-1 / m(H)}\right)^{-e\left(H^{\prime}\right)}\left(\frac{n}{h}\right)^{2 v(H)}\left(\frac{d}{n}\right)^{2 e(H)} p^{2 e(H)} \\
& \leqslant 2^{h} h^{h} C^{-1} n^{-1}\left(\frac{n}{h}\right)^{2 v(H)}\left(\frac{d}{n}\right)^{2 e(H)} p^{2 e(H)} .
\end{aligned}
$$

Thus, we see that

$$
\begin{aligned}
\frac{\mu^{2}}{2 \Lambda} & \geqslant \frac{\left(\frac{n}{2 h}\right)^{2 v(H)}\left(\frac{d}{n}\right)^{2 e(H)} p^{2 e(H)}}{2(2 h)^{h} C^{-1} n^{-1}\left(\frac{n}{h}\right)^{2 v(H)}\left(\frac{d}{n}\right)^{2 e(H)} p^{2 e(H)}} \\
& =\frac{C n}{2^{2 h+1}(2 h)^{h}} .
\end{aligned}
$$

Using Janson's inequality, we have

$$
\begin{aligned}
P(X=0) & \leqslant \exp \left(-\frac{\mu^{2}(1-\gamma)}{2 \Lambda}\right) \leqslant \exp \left(-\frac{C(1-\gamma)}{2^{2 h+1}(2 h)^{h}} n\right) \\
& \leqslant \exp \left(-\frac{C}{2^{2 h+2}(2 h)^{h}} n\right) .
\end{aligned}
$$

Since there are at most $2^{h n}$ ways to get $V_{1}, \ldots, V_{h}$, the probability that there is a coloring of $V(G)$ so that each color class is of size at most $n / 2 h$ and $G_{p}$ contains a rainbow copy of $H$, is at most

$$
2^{h n} \exp \left(-\frac{C}{2^{2 h+2}(2 h)^{h}} n\right)=\exp \left(h n \log 2-\frac{C}{2^{2 h+2}(2 h)^{h}} n\right)=o(1)
$$

provided $C \geqslant 2^{2 h+2}(2 h)^{h+1}$.

\section{$6 \quad H$-tiling}

Proof of Theorem 4. (i) Let $H^{\prime}=\left(V^{\prime}, E^{\prime}\right)$ be any subgraph of $H$ for which $e\left(H^{\prime}\right) /\left(v\left(H^{\prime}\right)-\right.$ $1)=m(H)$. Denote $e^{\prime}=e\left(H^{\prime}\right)$ and $v^{\prime}=v\left(H^{\prime}\right)$. Let $\mathcal{H}=\left\{H_{i}: i \in I\right\}$ denote the set of all labeled copies of $H^{\prime}$ in $G$. Write $X$ for the number of vertex-disjoint copies of $H^{\prime}$ in $G_{p}$.

It suffices to show that almost surely $X<(1-\epsilon) n / h$. We use the second moment 
method to do so. We first calculate the expectation of $X$.

$$
\begin{aligned}
E(X) & =(1+o(1)) \frac{n^{v^{\prime}}}{\operatorname{aut}\left(H^{\prime}\right)}\left(\frac{d}{n}\right)^{e^{\prime}} p^{e^{\prime}} \\
& =(1+o(1)) \frac{n^{v^{\prime}}}{\operatorname{aut}\left(H^{\prime}\right)}\left(\frac{d}{n}\right)^{e^{\prime}} c^{e^{\prime}} \frac{n^{e^{\prime}-e^{\prime} / m(H)}}{d^{e^{\prime}}} \\
& =(1+o(1)) \frac{c^{e^{\prime}}}{\operatorname{aut}\left(H^{\prime}\right)} n^{v^{\prime}-\left(v^{\prime}-1\right)} \\
& =(1+o(1)) \frac{c^{e^{\prime}}}{\operatorname{aut}\left(H^{\prime}\right)} n .
\end{aligned}
$$

Choosing an appropriate constant $c$, we have $E(X)<(1-\epsilon) n / 2$.

We next show that $\operatorname{Var}(X)=E\left(X^{2}\right)-\mu^{2}=o\left(\mu^{2}\right)$, which along with Chebyshev's inequality implies that $X$ is concentrated around its mean. If two copies $H_{i}, H_{j} \in \mathcal{H}$ share at least one edge in $G$, we denote $i \sim j$. Thus

$$
\begin{aligned}
E\left(X^{2}\right) & =\sum_{H_{i}, H_{j} \in \mathcal{H}} P\left(H_{i} \cup H_{j} \subseteq G_{p}\right) \\
& =\sum_{E\left(H_{i} \cap H_{j}\right)=\emptyset} P\left(H_{i} \cup H_{i} \subseteq G_{p}\right)+\sum_{i \sim j} P\left(H_{i} \cup H_{i} \subseteq G_{p}\right) \\
& =: t_{1}+t_{2},
\end{aligned}
$$

where the first sum $t_{1}:=\sum_{E\left(H_{i} \cap H_{j}\right)=\emptyset} P\left(H_{i} \cup H_{i} \subseteq G_{p}\right)$ is over ordered pairs that share no edges, and the second sum $t_{2}:=\sum_{i \sim j} P\left(H_{i} \cup H_{i} \subseteq G_{p}\right)$ is over all ordered pairs that intersect in at least one edge.

Clearly, if $H_{i}, H_{j} \in \mathcal{H}$ are edge-disjoint, then the events $H_{i} \subseteq G_{p}$ and $H_{j} \subseteq G_{p}$ are independent. Hence

$$
\begin{aligned}
t_{1} & =\sum_{E\left(H_{i} \cap H_{j}\right)=\emptyset} P\left(H_{i} \subseteq G_{p}\right) \cdot P\left(H_{i} \subseteq G_{p}\right) \\
& \leqslant \sum_{H_{i}, H_{j} \in \mathcal{H}} P\left(H_{i} \subseteq G_{p}\right) \cdot P\left(H_{i} \subseteq G_{p}\right)=E^{2}(X) .
\end{aligned}
$$

Now we estimate the sum over the ordered pairs that share at least one edge. The intersection of any $H_{i}$ and $H_{j}$ is a subgraph $H^{\prime \prime}=\left(V^{\prime \prime}, E^{\prime \prime}\right)$ of $H^{\prime}$, with $e\left(H^{\prime \prime}\right) \geqslant 1$. Thus we can partition the range of the second sum $t_{2}$ according to the various possible 


$$
\begin{aligned}
& H^{\prime \prime}=H_{i} \cap H_{j} . \\
& t_{2}=\sum_{H^{\prime \prime} \subseteq H^{\prime}} \sum_{H_{i} \cap H_{j} \simeq H^{\prime \prime}} P\left(H_{i} \cup H_{i} \subseteq G_{p}\right) \\
& \leqslant \sum_{H^{\prime \prime} \subseteq H^{\prime}} \frac{n^{v^{\prime}}}{\operatorname{aut}\left(H^{\prime}\right)}\left(\frac{d}{n}\right)^{e^{\prime}} p^{e^{\prime}} \cdot \frac{n^{v^{\prime}-v^{\prime \prime}}}{\operatorname{aut}\left(H^{\prime} \backslash H^{\prime \prime}\right)}\left(\frac{d}{n}\right)^{e^{\prime}-e^{\prime \prime}} p^{e^{\prime}-e^{\prime \prime}} \\
& =\sum_{H^{\prime \prime} \subseteq H^{\prime}} \frac{n^{2 v^{\prime}-v^{\prime \prime}}}{\operatorname{aut}\left(H^{\prime}\right) \operatorname{aut}\left(H^{\prime} \backslash H^{\prime \prime}\right)}\left(\frac{d}{n}\right)^{2 e^{\prime}-e^{\prime \prime}}\left(c \frac{n^{(m(H)-1) / m(H)}}{d}\right)^{2 e^{\prime}-e^{\prime \prime}} \\
& =\sum_{H^{\prime \prime} \subseteq H^{\prime}} \frac{c^{2 e^{\prime}-e^{\prime \prime}}}{\operatorname{aut}\left(H^{\prime}\right) \operatorname{aut}\left(H^{\prime} \backslash H^{\prime \prime}\right)} n^{2 v^{\prime}-v^{\prime \prime}-\frac{v^{\prime}-1}{e^{\prime}}\left(2 e^{\prime}-e^{\prime \prime}\right)} \\
& \leqslant \sum_{H^{\prime \prime} \subseteq H^{\prime}} \frac{c^{2 e^{\prime}-e^{\prime \prime}}}{\operatorname{aut}\left(H^{\prime}\right) \operatorname{aut}\left(H^{\prime} \backslash H^{\prime \prime}\right)} n^{2 v^{\prime}-v^{\prime \prime}-2\left(v^{\prime}-1\right)+\left(v^{\prime \prime}-1\right)} \quad\left(\frac{v^{\prime}-1}{e^{\prime}} \leqslant \frac{v^{\prime \prime}-1}{e^{\prime \prime}}\right) \\
& \leqslant 2\left(\begin{array}{c}
v^{\prime} \\
2
\end{array}\right) c^{2 e^{\prime}-e^{\prime \prime}} n=c_{1} n,
\end{aligned}
$$

where $c_{1}:=2^{\left(\begin{array}{c}v^{\prime} \\ 2\end{array}\right)} c^{2 e^{\prime}-e^{\prime \prime}}$. Hence

$$
\begin{aligned}
\frac{\operatorname{Var}(X)}{E^{2}(X)} & =\frac{E\left(X^{2}\right)-\mu^{2}}{\mu^{2}} \\
& \leqslant \frac{\mu^{2}+c_{1} n-\mu^{2}}{\mu^{2}}=O\left(\frac{1}{n}\right),
\end{aligned}
$$

and by Chebyshev's inequality,

$$
P(|X-E(X)| \geqslant E(X)) \leqslant \frac{\operatorname{Var}(X)}{\mu^{2}}=o(1) .
$$

Since $c$ can be small enough such that $E(X) \leqslant(1-\epsilon) n / 2 h$, it follows that

$$
P(X \geqslant(1-\epsilon) n / h) \leqslant P(|X-E(X)| \geqslant E(X))=o(1) .
$$

Thus we see that almost surely $X<(1-\epsilon) n / h$, in other words, there are less than $(1-\epsilon) n / h$ vertex-disjoint copies of $H^{\prime}$ in $G_{p}$. Hence there are less than $(1-\epsilon) n / h$ vertexdisjoint copies of $H$ in $G_{p}$.

(ii) It suffices to show that every subset of $\epsilon n$ vertices contains a copy of $H$. Lemma 9 will do the job. It shows that almost surely every subset of $\epsilon n$ vertices in $G_{p}$ contains a copy of $H$. Thus $G_{p}$ contains at least $(1-\epsilon) n / h$ vertex-disjoint copies of $H$.

The proof of Theorem 4 can be easily adapted to give a threshold function for the subgraph containment problem. Calculations are in much the same way as in the proof of Theorem 4 (i).

Analogously to Theorem 4, for a fixed graph $H$, define

$$
m^{\prime}(H):=\max \left\{\frac{e\left(H^{\prime}\right)}{v\left(H^{\prime}\right)}: H^{\prime} \subseteq H\right\} .
$$


Sketch of proof of Theorem 5. The proof is a direct application of the second moment method, and is similar to that of Theorem 4 (i).

Let $H^{\prime}$ be a subgraph of $H$ for which $e\left(H^{\prime}\right) / v\left(H^{\prime}\right)=m^{\prime}(H)$. Let $X$ and $X^{\prime}$ be the number of copies of $H$ and $H^{\prime}$ in $G_{p}$, respectively. For $p=c n^{\left(m^{\prime}(H)-1\right) / m^{\prime}(H)} / d$, it is easy to verify that $E\left(X^{\prime}\right) \rightarrow 0$ as $n \rightarrow \infty$, if $c$ sufficiently small.

For $p=C n^{\left(m^{\prime}(H)-1\right) / m^{\prime}(H)} / d$, use the second moment method to show that $\operatorname{Var}(X) / E^{2}(X) \rightarrow 0$ as $n \rightarrow \infty$, if $C$ is sufficiently large.

\section{Acknowledgements}

The author is indebted to an anonymous referee for many comments and suggestions, which are very insightful and helpful.

\section{References}

[1] N. Alon and J. Spencer, The Probabilistic Method, 2nd Ed., John Wiley, 2000.

[2] N. Alon and R. Yuster, Threshold functions for $H$-factors, in Combinatorics, Geometry and Probability, Cambridge University Press, Cambridge, 1997, 63-70.

[3] N. Alon and R. Yuster, $H$-factors in dense graphs, J. Combin. Theory Ser. B 66 (1996), 269-282.

[4] B. Bollobás, Threshold function for small graphs, Math. Proc. Cambridge Philos. Soc. 33, 476-484.

[5] B. Bollobás, Random Graphs, 2nd Ed., Cambridge University Press, New York, 2001.

[6] P. Erdős and A. Réyni, On the evolution of random graphs, Publ. Math. Inst. Hungar. Acad. Sci. 5 1960, 17-61.

[7] A. Frieze, M. Krivelevich, Hamilton cycles in random subgraphs of pseudo-random graphs, Discrete Math. 256 (2002), 137-150.

[8] A. Frieze,M. Krivelevich, R. Martin, The emergence of a giant component in random subgraphs of pseudo-random graphs, Random Structures Algorithms 24 (2004), 4250 .

[9] A. Hajnal and E. Szemerédi, Proof of a Conjecture of Erdős, In Combinatorial Theory and Its Applications, Vol. 2, North-Holland, 1970, 601-623.

[10] A. Johansson, J. Kahn and V. Vu, Factors in random graphs, Random Structures Algorithms 33 (2008), 1-28.

[11] M. Krivelevich and B. Sudakov, Pseudo-random graphs, in More Sets, Graphs and Numbers, Bolyai Society Mathematical Studies 15, Springer, 2006, 199-262.

[12] D. Kühn and D. Osthus, Embedding large subgraphs into dense graphs, in Surveys in Combinatorics 2009, Cambridge University Press, 2009, 137-167.

[13] T. Łuczak, A. Ruciński,B. Voigt, Ramsey properties of random graphs, J. Combin. Theory Ser. B 56 (1992), 55-68.

[14] J. Shen, Connectivity of random subgraphs of pseudo-random graphs, submitted. 